\title{
VIVE LE RAFALE, VIVE LA REPUBLIQUE, ET VIVE LA FRANCE1: RETOUR SUR LA PLACE DU MILITAIRE EN FRANCE
}

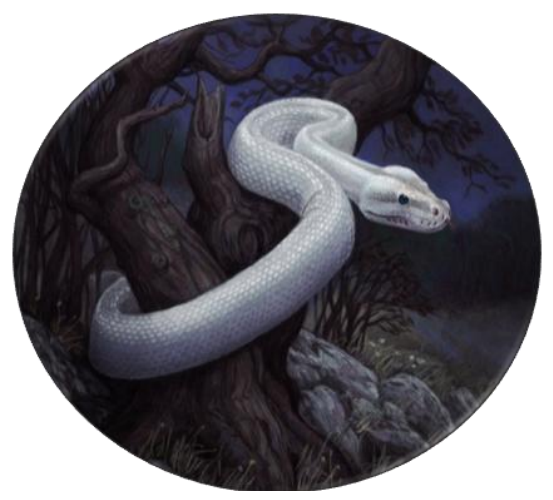

Claude Serfati

\section{Resume}

Une nouvelle conjoncture mondiale s'est mise en place à la fin des années 2000. Comme tout grand tournant dans l'histoire du capitalisme, celle-ci combine de façon originale des dimensions économiques et géopolitiques. Dans le contexte où la crise de 2008 continue d'exercer des effets sociaux ravageurs, des processus politiques et sociaux considérables ont profondément modifié la configuration géopolitique qui s'était mise en place après la disparition de l'URSS en 1991. Les gouvernements Sarkozy et Hollande ont saisi les opportunités créées par cette nouvelle conjoncture. Ils multiplient les interventions militaires en Afrique, où la France possède des intérêts économiques et politico-militaires primordiaux et promeuvent une intense diplomatie militaro-économique au Moyen-Orient. Cependant, l'interventionnisme militaire, que les gouvernements français tentent de faire soutenir par l'UE en l'utilisant comme 'un avantage comparatif vis-à-vis de leurs partenaires, est impuissant à arrêter ou même enrayer les conflits, et il place la France dans un engrenage dangereux.

\section{UNE NOUVELLE CONJONCTURE INTERNATIONALE S'EST OUVERTE A LA FIN DES ANNEES 2000}

Une conjonction de facteurs economiques et eeopolitiques

\footnotetext{
${ }^{1}$ François Hollande, lors de sa visite du site de Dassault aviation à Mérignac, 4 mars http:/ / www.elysee.fr/declarations/article/visite-du-site-de-dassault-aviationa-merignac-3/
} 
Une hypothèse constitutive de cet article est qu'un changement majeur dans la conjoncture économique et géopolitique mondiale a eu lieu à partir de la fin des années 2000. Les deux dimensions doivent être prises en compte car l'état de l'économie mondiale, en tant que totalité systémique, résulte de combinaison de la dynamique d'accumulation du capital, largement contrôlée par quelques centaines de grands groupes pour l'essentiel originaires des grands pays capitalistes développés, et de l'organisation du système interétatique. Du point de vue économique, la crise de 2008 s'est transformée en une crise qui se traduit par des niveaux de chômage inconnus depuis des décennies, et en Europe par une 'grande récession' dont même les responsables politiques admettent que pour y mettre fin, les politiques d'austérité sont une condition nécessaire, mais nullement suffisante2. L'intégration de la Chine à l'économie mondiale quelques années après la disparition de l'URSS (1991) a certes permis à l'accumulation du capital réalisés par les grands groupes, de retrouver une certaine vigueur pendant une dizaine d'années. Une conséquence est que depuis la crise de 2008, l'économie mondiale est encore plus dépendante de l'état de l'économie chinoise, qui est scruté avec beaucoup d'inquiétudes. Car si la croissance de l'économie mondiale dépend de celle de la Chine, l'accumulation du capital en Chine dépend dans une large mesure de l'état de l'économie mondiale. En Chine, les surcapacités de production qui s'accumulent dans les secteurs de la construction, des équipements industriels, des mines s'accompagnent sans surprise d'une baisse du taux de rentabilité des grandes entreprises, lourdement endettées3. La prospérité du capital financier, au sens de capital porteur de revenus financiers (dividendes, intérêt, royalties, etc.), globalement renforcée depuis la crise de 2008 produit des conséquences qui s'entrelacent avec le ralentissement de

2 Wall Street Journal, «Q\&A: ECB President Mario Draghi", 23 février 2012 et plus récemment The Economist, « TakingEurope's pulse ", 7 mai 2015.

${ }^{3} \mathrm{IMF}, 2015$, p.38. 
l'accumulation du capital. En D'où le diagnostic établi par F. Chesnais il y a plus de trois ans «qu'aucune «sortie de crise» ne se dessine pour le capital au plan mondial, dans un horizon de temps prévisible»4.

Sur le plan géopolitique, à la fin des années 2000, trois facteurs majeurs ont mis fin à la situation qui avait été créée par la disparition de l'URSS. L'affaiblissement de la domination politique des Etats- Unis en constitue le trait le plus spectaculaire. Le coût économique des guerres en Irak et en Afghanistan est colossal5 et la contribution des dépenses engagées pour ces guerres à l'aggravation du déficit budgétaire des Etats-Unis qu'elles provoquent est significative, même si ce n'est pas la plus importante6. Le coût géopolitique est plus élevé encore. Ces guerres ont créé des ondes de choc incontrôlées au Moyen-Orient et en Afrique où elles ont aggravé le chaos qui existe depuis des dizaines d'années dans certains pays. De plus, la décision de l'Administration Busha produit ce résultat remarquable de faciliter l'émergence de l'Iran comme puissance régionale, avec laquelle les Etats-Unis doivent aujourd'hui composer7.

La situation actuelle dément donc totalement les prévisions de domination de l' «empire » qui avaient été formulées par ses thuriféraires mais aussi par ses critiques, au lendemain de la disparition de l'URSS. Pour les néoconservateurs, la disparition de l'USS devait consolider l'hégémonie

${ }^{4}$ F.Chesnais, «Aux racines de la crise économique mondiale», 3 janvier 2012, http://alencontre.org/economie/aux-racines-de-la-crise-economique-mondial e.html

${ }^{5}$ Voir l'ouvrage de J. Stiglitz et L. Bilmes, The Three Trillion Dollar War, Penguin Books, 2008. A la fin juin 2015, le coût total était estimé à 4,4 trillion de dollars, voir http:/ / costsofwar.org/

6Sur la période 2009-2019, les conséquences de la récession de 2008 et des mesures budgétaires prises pour y faire face comptent pour $44 \%$ du déficit budgétaire, les réductions d'impôts prises sous la présidence Bush $41 \%$, et le coût des guerres 15\%, Center on Budget and Policy Priorities « Economic Downturn and Legacy of Bush Policies Continue to Drive Large Deficits », 28 février 2013.

7Voir G. Friedman, "Strategic Reversal: The United States, Iran, and the Middle East Analysis", 24Novembre 2014 
des Etats- Unis pendant des décennies, facilitant l'imposition de la 'démocratie' grâce à l'usage de la puissance militaire (le nation-building) dont le renversement de S. Hussein était la première grande étape. Selon eux, la guerre en Irak serait évidemment de courte durée et d'un faible coût8. Du côté des critiques de la politique américaine, y compris marxistes, le qualificatif d"empire' fut également abondamment utilisé au début des années 2000, car il semblait le mieux refléter la mise sous tutelle par les Etats-Unis de l'ensemble de la planète,y compris des autres pays impérialistes (un terme jugé par contre démodé).Les débats qui eurent lieu à cette époque9 révèlent égalementl'erreur d'appréciation qui fut faite sur la capacité des Etats-Unis à conforter leur domination. Car les Etats- Unis, bien que bénéficiaires des transformations économiques et géopolitiques des années 1990, n'étaient pas 'hors la crise’ et leurs dirigeants n'avaient ni les moyens, ni la volonté de gérer les convulsions planétaires produites par la disparition de l'URSS et le développement fortement inégal provoqué par la mondialisation du capital. Dans ce cadre analytique, il était donc possible d'affirmer avant qu'elle fut mise en œuvre que la décision prise par G.W. Bush de mener la guerre en Irak possédait un niveau élevé d'aventurisme, au sens définitionnel de comportements dont le sujet ne maitrise pas les conséquences10.

Aujourd'hui, une erreur symétrique à celle qui fut faite par l'assimilation des Etats-Unis à un empire 'informel' mais sans limite serait de négliger le poids de la puissance politico-militaire dans les décisions de l'Administration. Le partenariat transpacifique (TPP) vise parmi d'autres

8Par exemple Lindsay, le conseiller du ministre de la défense Rumsfeld, avançait un coût de 100 à 200 milliards de dollars maximum, Elisabeth Bumiller «Threats And Responses: The Cost; White House Cuts Estimate Of Cost Of WarWith Iraq, New York Times, 31Decembre 2002.

9 Voir par exemple le débat dans ATTAC, dans Carré rouge et sur le site A l'encontre.

${ }^{10} C^{\prime}$ est cette position que F. Chesnais et moi avons défendue à l'époque. 
objectifs, à 'contenir' les ambitions chinoises par des moyens économiques et militaires, il nourrit la militarisation des alliés régionaux des Etats-Unis, et de façon significative celui du Japon. Les documents stratégiques rappellent que $60 \%$ des forces armées américaines doivent être basées dans la région Asie-Pacifique et les ventes d'armes américaines aux Etats de la région accompagnent la hausse considérable des budgets militaires. Les visées militaires sont à ce point patentes que des dirigeants de pays Asiatiques(en particulier l'Australie, la Nouvelle-Zélande et la Malaisie) ont fait part de leurs inquiétudes quant à l'utilisation du TTP contre la Chine11.

Les fissures du système inter-étatique, qui ébranlent sérieusement l'édifice, constituent un deuxième facteur dans l'avènement de la nouvelle conjoncture internationale. La domination solidement ancrée du capital financier et la mise en œuvre des politiques d'ajustement structurel ont accéléré l'implosion de dizaines d'Etats, qualifiés d' "Etats faillis » par le Département d'Etat des Etats-Unis, depuis 2001, d'« Etats sous tension » par la Banque mondiale et d' "Etats fragiles » par l'OCDE qui en dénombrait 51 en 201312. Or, la 'forme Etat' demeure absolument indispensable pour la reproduction des rapports sociaux fondés sur l'exploitation et la domination, et les pays dominants de la planète prennent soin que tous les Etats, y compris ceux déclarés 'faillis', bénéficient de la légitimité auprès de leurs populations grâce à leur statut de membres de la 'communauté internationale' (l'ONU).

Enfin, la situation géopolitique est totalement modifiée depuis 2011 à la suite du mouvement révolutionnaire qui a renversé la dictature de Ben Ali et dont l'écho a été entendu dans d'autres pays d'Afrique et du

11 Public Citizen, Memo To Reporters, April 28, 2015.

${ }^{12}$ L'OCDE note que 26 des 51 'Etats fragiles' appartiennent aux pays à 'revenus moyens supérieurs'. L'implosion étatique ne concerne donc plus seulement la catégorie des pays 'laissés pour compte', OECD, Fragile States 2014 : Domestic Revenue Mobilisation in Fragile States, Paris, 2014. 
Moyen-Orient. Les mobilisations de millions de personnes dans les rues aboutissent au renversement ou tout au moins à l'affaiblissement des régimes dont la longue existence a reposé sur le soutien des pays dominants, et dont la survie en dépend encore plus. La décomposition accélérée d'un certain nombre d'entre eux requiert une ingérence militaire directe des pays dominants, c'est particulièrement le cas en Afrique.

\section{La France saisit les opportunites}

Pendant la période de guerre froide, la diplomatie gaulliste a adopté une position d'autonomie vis-à- vis des Etats-Unis. Il ne s'agissait pas d'un comportement superficiel ou d'un trait personnel du fondateur de la $\mathrm{V}^{\circ}$ république, qui était au contraire convaincu que la France pouvait exploiter l'antagonisme Est-Ouest et jouer, à sa juste place, un rôle dans la défense de l'ordre mondial. Les relations entre les Etats-Unis et la France n'excluaient donc pas les désaccords, mais dans un cadre qui était communément accepté. En plus du statut conféré par l'arme nucléaire et le siège de membre permanent au Conseil de sécurité, les ambitions gaullistes étaient essentiellement centrées sur l'Afrique, et les Etats-Unis acceptaient cette vision. Ainsi, l'Administration américaine a traditionnellement considéré l'Afrique comme procédant «d'une responsabilité Européenne particulière» de la même façon que les Européens doivent reconnaitre «la responsabilité américaine en Amérique latine», selon les termes d'un membre de l'Administration Kennedy ${ }^{13}$. La position de l'Administration Clinton fut à peu près la même, d'autant plus que le retrait catastrophique des forces américaines de Somalie en octobre 1993a créé un traumatisme durable.

${ }^{13}$ G. Ball, sous-secrétaire d'Etat, cité dans Peter J. Schraeder, “Cold War to Cold Peace: Explaining U.S.-French Competition in Francophone Africa", Political Science Quarterly, Vol. 115, No. 3, 2000, pp. 395-419. 
La position hostile à la guerre des Etats-Unis en Irak qui a été défendue par D. de Villepin à l'ONU en 2003, qui correspondait d'ailleurs à une position très majoritaire à l'échelle mondiale ${ }^{14}$, s'inscrit dans cette tradition d'autonomie de la diplomatie française. Elle constituait donc un important correctif aux années 1990 au cours desquelles la France avait contribué à son propre affaiblissement au Moyen-Orient en participant à la guerre contre S. Hussein, qui avait été depuis les années 1970 'notre meilleur ami'.

L'impasse politique dans laquelle les Etats-Unis se sont trouvés à la suite de l'invasion de l'Iraq a donc ouvert une fenêtre d'opportunité à la France et facilité quelques années plus tard l'activisme militaro-diplomatique de la France en Afrique. La France touche en quelque sorte les dividendes de la position défendue en 2003 par D. de Villepin à l'ONU. A la fin des années 2000, la France était en effet, au sein du 'bloc transatlantique"15, la mieux à même de prendre les initiatives destinées à faire face au chaos qui résultait de l'effondrement des appareils d'Etat de plusieurs pays d'Afrique. On peut même affirmer sans erreur qu'elle était la seule à pouvoir le faire. En effet, les deux autres grandes puissances militaires occidentales n'ont aujourd'hui pas la capacité et la volonté de prendre une part active en Afrique : les Etats-Unis peinent à sortir de leur enlisement au Moyen-Orient et le Royaume-Uni, dont le désengagement militaire en Afrique est ancien, est encore secoué par l'implication du gouvernement Blair dans la guerre en Irak. De plus, les militaires et les services de renseignements officiels et parallèles français

${ }^{14}$ Ewen MacAskill and Julian Borger, «Iraq war was illegal and breached UN charter, says Annan », The Guardian, 16 septembre 2004.

15J'ai proposé en 2003, alors que 1"'unilatéralisme' des Etats-Unis atteignait son apogée, la notion de bloc transtlantique hiérarchisé comme cristallisation de la domination des Etats-Unis et de leurs alliés sur la planète. Pour une actualisation, C. Serfati, "The transatlantic bloc of states and the political economy of the Transatlantic Trade and Investment Partnership", Work Organisation, LabourEGlobalisation, Vol. 9, No. 1, Spring 2015. 
disposent d'une connaissance du terrain inégalé, ce qui est reconnu même par les militaires Américains (voir plus loin) ${ }^{16}$. L'Administration et l'Etat-major américains placent manifestement une grande confiance dans l'engagement militaire de la France ${ }^{17}$.

\section{Les enjeux de la presence française en Afrique}

En réalité, les interventions militaires de la France en Afrique résultent d'un ensemble de facteurs convergents, et ilfaut se garder de toute recherche monocausale. Les intérêts économiques et géopolitiques enracinés depuis des décennies fournissent une trame explicative sur laquelle d'autres éléments viennent s'accrocher. On peut citer l'influence exercée dans la vie politique et médiatique par des fractions du patronat très implantées en Afrique, l'importance pour les militaires de la forte présence des armées dans les anciennes colonies sub-sahariennes qui permet d'amoindrir le traumatisme créé par les défaites en Indochine et en Algérie, ou encore les choix propres des Présidents de droite puis de gauche dont elle constitue le 'domaine réservé', une notion qui n'a pas de fondement juridique, mais qui est conforme aux institutions de la $\mathrm{V}^{\circ}$ république. On mesure donc l'épaisseur sociale et politique de ce qui est parfois nommé lafranceafrique, trop souvent réduite à des réseaux quasi-conspiratifs dont l'acte de décès est périodiquement annoncé.

16 Selon un haut fonctionnaire en charge des questions africaines, la région subsaharienne est «l'une des rares

- sinon la seule - où l'expertise de la France est plus importante que celle des États-Unis ", " 'Hollande l'Africain'? La politique africaine de la France à la croisée des chemins. Interview de Yves Gounin", Actuelle de l'Ifri, septembre 2012,

http:/ /ifri.labinnovation.fr/fr/publications/editoriaux/lafrique-questions/laf rique-questions- ndeg13-hollande-lafricain-politique-o

17 Defense News, "France Displaces Britain as Key US Military Ally", 19 mars 2015. 
En tout état de cause, l'intensification des interventions militaires n'a rien de surprenant, même si elle a semé le trouble y compris dans «la gauche de la gauches ${ }^{18}$. Le Livre blanc sur la défense et la sécurité nationale de 2013rappelle que «Le Sabel, de la Mauritanie à la Corne de l'Afrique, ainsi qu'une partie de l'Afrique subsaharienne - notamment le Golfe de Guinée et les pays riverains sont des zones prioritaires pour la France : le Livre blanc 2013 se distingue en particulier par la place accordée au continent africain ... [De plus] La sécurité de la zone qui s'étend des rives de la Méditerranée orientale au Golfe Arabo-Persique constitue une priorité en soì ${ }^{19}$.Ce recentrage sur la zone de présence traditionnelle de la France grâce au désengagement d'Afghanistan est selon des militaires, une source d'efficacité opérationnelle accrue ${ }^{20}$.

Cette section rappelle donc l'importance économique de l'Afrique pour la France et elle prend le contrepied des thèses 'déclinistes' qui suggèrent un désintérêt, voire un désengagement des grands groupes français. Elle analyse ensuite l'importance du continent du point de vue militaire, et l'expérience majeure que les armées françaises tirent de leur présence active dans les conflits certes différents des guerres inter-impérialistes, et qui ont évidemment valeur d'enseignement pour d'autres conflits qui ont lieu dans le monde.

18 Voir Jean Batou, « Afrique : Redéploiement de l'impérialisme français et sidération humanitaire de la gauche $\gg$,Contretemps, http://www.contretemps.eu/interventions/afrique-red\%C3\% A9ploiementimp\%C3\%A9rialisme-fran\%C3\%A7ais-sid\%C3\%A9ration-humanitaire-gauche d

19P.13. Voir également le rapport 'bi-partisan' « Sahel : Pour une approche globale », Rapport d'information $\mathrm{n}^{\circ}$

(2012-2013) de MM. Jean-Pierre Chevènement et Gérard Larcher, fait au nom de la commission des affaires étrangères, de la défense et des forces armées ", Sénat, 3 juillet 2013.

20 O. Tramond, P. Seigneur, "Operation Serval Another Beau Geste of France in Sub-Saharan Africa?", MILITARY REVIEW, Novembre-Décembre 2014. 


\section{A la recherche du 'miracle' africain}

Dans le contexte où les effets de la crise ouverte en 2008 continuent d'exercer leurs effets dévastateurs à l'échelle mondiale, les annonces répétées d'un 'miracle Africain' visent à apaiser les craintes. Le principal signe auxquels se raccrochent les discours est celui d'un taux de croissance du PIB supérieur à celui de l'économie mondiale depuis le début de la décennie 2000. Pourtant, le continent Africain n'a pas globalement modifié sa place en tant que territoire d'accueil des Investissements directs à l'étranger (IDE) effectués par les sociétés transnationales au cours des années 2000, sa part reste obstinément en-dessous de 3\% du stock total d'IDE, elle a même été inférieure en 2013 à celle des années 1990-1995. De plus, le nombre de projets d'IDE 'créateurs de capacités de production' (greenfield), qui ont, pour les pays d'accueil, des effets en principe plus bénéfiques que les IDE destinés à racheter des entreprises existantes (en général des entreprises publiques au moment de leurs privatisations) étaient en 2013 inferieurs au nombre de projets réalisés en 2003 respectivement de $1 \%$ dans les pays développés, de $6 \%$ dans les pays en développement et de $12 \%$ en Afrique21.

En réalité, la croissance du PIB, très différenciée selon les pays et très souvent même à l'intérieur des pays, est extrêmement fragile, elle repose sur des choix sélectifs opérés par les grands groupes industriels et financiers. Elle est accompagnée sur le continent Africain d'une diminution du secteur manufacturier dans la valeur ajoutée au cours de la décennie 2000. Au cours des années 2000-2011, la part de la valeur ajoutée dans le PIB est tombée à $11 \%$ alors qu'elle était de $14 \%$ au cours des années

${ }^{21}$ UNCTAD, 2014, Web table 19. "Value of greenfield FDI projects, by destination, 2003-2013". 
1990-199922. La croissance macroéconomique repose sur une extraversion renforcée des productions agricoles et un déficit croissant dans la balance commerciale des biens alimentaires. L'Afrique exporte des produits agricoles mais importe massivement son alimentation, en particulier des céréales pour nourrir sa population23.

L'Afrique subsaharienne souffre également l'emprise du capital financier. Les institutions financières internationales se félicitent que les pays de la région subsaharienne aient accédé aux marchés financiers internationaux pour l'émission de bons du trésor, ils y voient les promesses d'un développement puissants. En fait, les gouvernements émettent des titres de la dette qui sont considérés comme «spéculatifs » par les agences de notation. Cette très médiocre notation mérite récompense : les investisseurs financiers prêtent donc à des taux d'intérêt très élevés, qui se situent toujours à plus de 3\% (300 points de base, disent les spécialistes) au-dessus du taux interbancaire de référence (LIBOR), et parfois beaucoup plus haut (7\% au-dessus du LIBOR) pour le Sénégal24. Ces exigences des prêteurs s'accompagnent de programmes de compression des déficits publics qui visent à juguler l'augmentation de la dette publique, consécutive à la montée des intérêts payés25, sans compter les risques de changes élevés - on peut dire inévitables - pour les pays emprunteurs puisque ils émettent leurs emprunts en dollars26.

22UNCTAD, The Economic Development in Africa Report 2014, Geneva.

${ }^{23}$ M. A. Rakotoarisoa, Ma. Iafrate, M.Paschali "Why has Africa become a net food importer? Explaining Africa agricultural and food trade deficits", Food and agriculture organization of the United Nations Rome 2011

${ }^{24}$ Un taux d'intérêt de 7\% double la somme initiale à rembourser en environ 8 ans.

${ }^{25}$ Entre 2012 et 2015 (prévision), le montant du service de la dette en proportion du PIB passera de 9,6\% en 2012 à 11,8\% en 2015.

${ }^{26} \mathrm{Un}$ rapport évalue les pertes qui résulteraient d'une dévaluation des monnaies nationales par rapport au dollar à un montant équivalent à 1,13\% du PIB des pays de la région, Judith E. Tyson, " Sub-SaharanAfrica International 
Il est vrai que la constitution de foyers d'accumulation du capital productif principalement dans les industries extractives, produit une concentration ouvrière, qui se combine avec les mouvements populaires conduits contre les gouvernements. La recension établie par les agences de presse AFP et Reuters indique une progression continue depuis 2000 des mouvements de protestation dans 52 pays d'Afrique, avec en tête les actions pour les augmentations de salaire27.

\section{Une importance persistante pour les entreprises françaises}

Il ne manque pas de discours qui soulignent le déclin des positions des intérêts économiques de la France en Afrique. Cet affaiblissement reflète la détérioration plus générale de l'industrie française sur de très nombreux marchés mondiaux, elle profite surtout à la Chine dont la progression des positions sur le continent est impressionnante. Cependant, la France bénéficie sur le continent de deux atouts précieux et à ce jour inégalés: d'une part un enracinement parfois séculaire de groupes agro-alimentaires, manufacturiers et financiers et d'autre part la proximité des dirigeants de la France et de ceux de ses anciennes colonies qui lui donne un 'droit spécial' d'ingérence militaire lorsqu'elle celle-ci correspond à des intérêts stratégiques.

Ces dimensions historiques, économiques et politiques totalement entrelacées expliquent deux évolutions significatives. D'abord, au cours de la période 2000-2013, les IDE réalisés par les entreprises Françaises en Afrique ont progressé deux fois plus vite que leurs IDE totaux ${ }^{28}$. Ensuite, la part des pays Africains dans le total des IDE français a triplé, passant de

Sovereign Bonds. Part II : Risks for Issuers », Overseas Development Institute, Janvier 2015.

${ }^{27}$ AFDB (avec l'OCDE et Le PNUD) ,African Economic Outlook, 2014.

${ }^{28}$ Ils ont été multipliés par 6,6 contre 3,4 pour les IDE totaux réalisés en Afrique. Source : base de données CNUCED. 
1,5\% à 4,6\% (tableau 1), Ceci sans compter les investissements réalisés sur place par les filiales de droit national des groupes français ${ }^{29}$.

\begin{tabular}{|l|l|l|}
\hline Part de la région dans le total des IDE sortants de France & $\mathbf{2 0 0 0}$ & $\mathbf{2 0 1 3}$ \\
\hline Zone Franc & 0,5 & 0,9 \\
\hline Afrique & 1,5 & 4,6 \\
\hline
\end{tabular}

Tableau 1: Evolution (2000-2013) des IDE français en Afrique et de la zone Franc en proportion des IDE français totaux

Source : Auteur, partir de la base de données de la CNUCED.

On peut donc parler d'une consolidation des positions française sur le continent Africain, puisque celui-ci occupe une part croissante dans les IDE français. Cette consolidation des intérêts économiques français s'est traduite par une forte pénétration hors de la zone franc (tels que l'Angola, le Nigéria, l'Afrique du sud) mais elle ne s'est pas faite au détriment de celle-ci (tableau 1). En somme, les fortes positions occupées dans les anciennes colonies (zone franc) ont été utilisés par les grands groupes français comme une rampe de lancement pour la conquête de nouveaux marchés.

${ }^{29}$ Ainsi que le Conseil des Investissements en Afrique (CIAN) le rappelle, "la spécificité de la présence française en Afrique est l'implantation locale or les investissements réalisés par les filiales de droit local ne sont pas comptabilisés dans ce stock », Rapport 2015, Les entreprises françaises et l'Afrique, Le MOCI, Hors-série, Décembre 204, p.12. 


\begin{tabular}{|c|c|c|c|c|c|c|c|c|}
\hline & $\begin{array}{l}\text { Effectif } \\
\text { s } \\
\text { (millier } \\
\text { s) }\end{array}$ & $\begin{array}{l}\text { en } \\
\%\end{array}$ & $\begin{array}{l}\text { Chiffre } \\
\text { d'affaire } \\
\text { s } \\
\text { (en } \\
\text { milliard } \\
\text { s) }\end{array}$ & $\begin{array}{l}\text { en } \\
\%\end{array}$ & $\begin{array}{l}\text { Nombr } \\
\text { e de } \\
\text { filiales }\end{array}$ & $\begin{array}{l}\text { en } \\
\%\end{array}$ & $\begin{array}{l}\text { Effectif } \\
/ \\
\text { filiales }\end{array}$ & $\begin{array}{l}\text { CA par } \\
\text { filiales } \\
\text { (millio } \\
\text { ns } \\
\text { d'euros } \\
\text { ) } \\
\end{array}$ \\
\hline $\begin{array}{l}\text { Maghreb } \\
\text { et Moyen- } \\
\text { Orient }\end{array}$ & 225,2 & 4,8 & 35,8 & 3,3 & 1751 & 5,6 & 128,6 & 20,4 \\
\hline $\begin{array}{l}\text { Afrique } \\
\text { francopho } \\
\text { ne }\end{array}$ & 80,5 & 1,7 & 14,1 & 1,3 & 533 & 1,7 & 151,1 & 26,5 \\
\hline $\begin{array}{l}\text { Afrique } \\
\text { non } \\
\text { francopho } \\
\text { ne }\end{array}$ & 55,8 & 1,2 & 13,1 & 1,2 & 486 & 1,6 & 114,7 & 27,0 \\
\hline Total & 361,5 & 7,7 & 63,0 & 5,8 & 2770,2 & 8,9 & 394,4 & 73,9 \\
\hline $\begin{array}{l}\text { Ensemble } \\
\text { Hour } \\
\text { Union } \\
\text { européenn } \\
\text { e }\end{array}$ & 2673,2 & 57,2 & 549,5 & $\begin{array}{l}51, \\
0\end{array}$ & 15130 & 48,8 & 176,7 & 36,3 \\
\hline $\begin{array}{l}\text { Ensemble } \\
\text { des } \\
\text { régions }\end{array}$ & 4671,2 & $\begin{array}{l}100 \\
0\end{array}$ & 1077,7 & 100 & 31004 & $\begin{array}{l}100, \\
0\end{array}$ & 150,7 & 34,8 \\
\hline
\end{tabular}

Tableau 2 : Implantation des groupes français internationalisés par grandes zones géographiques

Source : enquête FATS 2012

Les données fournies dans cet article indiquent que l'objectif a été tenu. Des propositions d'étendre la «Zone CFA» aux pays limitrophes pour en faire un bloc régional sont mêmes faites dans plusieurs rapports 30 .

On constate une polarisation des IDE français vers l'Afrique et le Moyen-Orient encore plus forte lorsque l'on s'intéresse aux secteurs de l'industrie et de la distribution (donc hors secteur financier). Les effectifs employés et le chiffre d'affaires réalisés par les groupes français présents dans ces deux régions représentent plus de 10\% de leurs emplois et chiffre d'affaires à l'étranger (hors UE).

30Par exemple, H. Védrine et alii, « Un partenariat pour l'avenir : 15 propositions pour une nouvelle dynamique économique entre l'Afrique et la France », Rapport au Ministre de L'économie et des Finances, Décembre 2013 
De fait, de nombreux groupes français, solidement implantés depuis des décennies et dont la sécurité est assurée par la présence de l'armée française, considèrent l'Afrique comme une base majeure de leur activités. Un rapport rédigé par des hommes d'affaires rappelle que, du point de vue du financement, «ils n'ont que l'embarras du choix [...] : AFD, BEI, Caisse de dépôts et de consignations (CDC), Banque mondiale, Exim Bank, AMIDA, etc.»31.

L'intérêt économique de l'Afrique est encore plus évident lorsqu'on observe les secteurs et les groupes industriels concernés. Il est difficile d'imaginer ce que serait le capitalisme français sans la présence du groupe Total (et naguère ELF) qui assure 30\% de sa production pétrolière grâce à l'Afrique, ou sans celle d'Aréva, dont la présence au Niger est une des causes de l'intervention française au Mali fin 2012. Ce ne sont pas les seuls groupes à avoir construit leur puissance grâce à leur présence en Afrique. En 2013, le groupe Bolloré a réalisé 21\% de son chiffre d'affaires en Afrique, soit plus que dans l'UE (hors France), ce qui est assez rare pour un groupe français d'envergure mondiale. Les bénéfices réalisés par le groupe Bolloré reposent essentiellement (par exemple à 90\% en 2013) sur ceux réalisés par ses activités en Afrique. La filiale africaine du groupe Bolloré (Bolloré Africa Logistics) revendique la première place dans le transport et de la logistique en Afrique, avec un réseau de 250 filiales et 24 000 employés dans 46 pays, soit l'essentiel de sa présence internationale (55 pays)32. L'activité du groupe Orange (ex France-Télécom), dont l'histoire et le positionnement de marché sont pourtant très différents de Bolloré, repose également fortement sur l'Afrique et le Moyen-Orient. En 2013, le groupe a réalisé la moitié de son chiffre d'affaires en France, et dans les

31Institut Montaigne, « Afrique-France : mettre en pratique le co-développement ", Contribution au XXVIe sommet Afrique-France, Décembre 2013, p.22.

32Bolloré, Rapport d'activité 2013. 
activités 'Reste du monde', l'Afrique et le Moyen-Orient ont représenté 51\% de l'activité (dont 15\% en Egypte).

Un autre aspect, souvent négligé, reflète l'importance de l'Afrique pour le capitalisme français. Le nombre d'entreprises françaises exportatrices vers le continent est disproportionné par rapport au volume des échanges. Alors que les flux d'exportations vers ce continent ont compté pour 6\% des échanges totaux de la France en 2013, le nombre d'entreprises françaises s'est élevé à un peu plus de 4000033 sur un total de 120700 entreprises exportatrices. Pour comparaison, le nombre totald'entreprises exportatrices vers l'Asie atteignait un peu moins de 30000, alors que le part de ce continent dans les exportations françaises a représenté 12,6\% des exportations totales de la France. On voit donc que le continent nourrit l'activité d'une partie importante des PME exportatrices françaises, ce qui révèle au-delà des échanges commerciaux, une densité de liens sociaux entre la métropole et les anciennes colonies.

\section{Lorsque 'marches' et conflits armes font bon ménage}

La consolidation des intérêts économiques de la France en Afrique ne dément pas seulement les discours 'déclinistes' sur l'importance du 'pré carré' africain. Elle pose la question des relations entre cette consolidation et l'abondance des conflits armés que connait le continent. La « mondialisation- réellement-existante » fonctionne aux antipodes des modèles élaborés par l'économie orthodoxe. La liberté de circulation des capitaux et des marchandises (mais pas celle des êtres humains) doit

33 Douanes, «Les opérateurs du commerce extérieur de la France. Données et tableauxAnnée 2014 - résultats

provisoires " douane

http://lekiosque.finances.gouv.fr/etudes/thematiques/A2014_operateurs_prov.pdf 
conduire à la paix et à la démocratie. Version contemporaine du 'doux commerce' espéré par Montesquieu, cette thèse au format 'pdf(pour peace-democracy- free markets) qui fut énoncée après la disparition de l'URSS est contredite depuis plus de vingt ans. L'accumulation de conflits armés 'localisés' dans les pays ‘périphériques' des pays développés, puis des conflits impliquant directement des armées de pays développés (guerres en Afghanistan et en Irak, en Yougoslavie) a même accéléré depuis 2011 (Libye, Mali, RCA, Ukraine). Le nombre total de conflits est resté à peu près le même depuis le début des années 2000, oscillant entre 33 et 37 par an. Les dirigeants des grands groupes ne sont d'ailleurs pas dupes, qui placent pour la première fois depuis dix ans les conflits géopolitiques au rang le plus élevé des risques les plus élevés à court terme et même dans les dix prochaines années encourus par le business ${ }^{34}$.

L'Afrique est particulièrement concernée par les conflits armés. La Banque mondiale, en se fondant sur la base de données de Uppsala Conflict Data Program (UCDP), signale qu'en 2013, il y avait 13 conflits armés 'intra-étatiques', mais qui impliquaient des réseaux internationaux (y compris d'autres gouvernements), un nombre qui a doublé depuis 2005, le point le plus bas depuis la disparition de l'URSS (AFDB, 2014,p.113) ${ }^{35}$. Néanmoins, les évolutions des IDE décrites depuis 2000 le montrent, les groupes français, pas plus que leurs homologues étrangers, ne sont découragés par le chaos provoqué par les guerres. Le comité qui regroupe les entreprises françaises en Afrique (CIAN) nous explique pourquoi : « Les grandes entreprises - Total, Carrefour, Bolloré, Société générale ou Somdiaa -, qui ont leurs aises à l'international et fréquentent l'Afrique de longue date, [...] savent "transformer le risque en opportunités". Elles ne se laissent pas impressionner par l'absence de visibilité politique, juridique et sociale, qui est le lot de nombreux pays

${ }^{34}$ World Economic Forum, Global Risks 2015, 10th Edition, Geneva.

${ }^{35}$ Centre de Développement de 1'OCDE, African Economic Outlook 2014. Global Value Chains and Africa's Industrialisation, Paris, 2014. 
africains, car elles ont appris que cette opacité - et les risques qui en découlent - est "compensée par un retour sur investissement élevé"136.

Après plus de trois décennies de conflits armés qui sont pérennes pour nombre d'entre eux, la position défendue par la Banque mondiale jusqu'au début des années 2000 n'est donc plus tenable. Selon les analyses de ses économistes ${ }^{37}$, les 'guerres civiles' reflétaient une 'mauvaise gouvernance' et plus généralement l'absence d'adhésion aux règles qui fondent la mondialisation : ouverture des marchés, démocratie et paix. A l'inverse de ces thèses, les guerres pour les ressources sont une composante de la mondialisation ${ }^{38}$. L'exploitation des ressources ne serait souvent pas possible sans les technologies et les compétences des grands groupes occidentaux. Les transferts d'argent par les grands groupes et les élites locales sont organisés avec le soutien des grandes banques multinationales ${ }^{39}$. De plus, ces guerres sont connectées aux pays développés par plusieurs canaux et acteurs : leurs gouvernements (en particulier par les détournements de fonds vers les partis politiques) ${ }^{40}$, leurs places financières via le recyclage de l'argent tiré de la prédation des ressources minérales vers

${ }^{36}$ Cité dans Alain Faujas, « Afrique-France : place au business » Jeune Afrique, vendredi 6 février 2015.

37 Par exemple, dans un article qui provoqua un large débat, Paul Collier and Anke Hoeffler, "Greed and Grievance in Civil Wars," Oxford Economic Papers, Vol. 56, No. 4, 2004, pp. 563-595.

38 A. Aknin et C. Serfati, "Guerres pour les ressources, rente et mondialisation ", Mondes en développement, 2008/3, $\mathrm{n}^{\circ} 143$.

${ }^{39}$ Lee A. Sheppard, une spécialiste des questions de 'prix de transfert' qui permettent aux grands groupes multinationaux d'échapper aux impôts (mais également de recycler des capitaux 'sales') est très critique contre les initiatives de l'OCDE «L'objectif du traité proposé par l'OCDE était de rendre la vie plus facile aux firmes multinationales allemandes, américaines, britanniques et françaises en s'assurant que la taxation de leur opérations hors de leur pays d'origine soit limitée, grâce au concept d'établissement permanent et une comptabilité séparée ", "Is Transfer Pricing Worth Salvaging?, Tax Notes, 30 juillet 2012, p.467.

${ }^{40}$ Voir par exemple les déclarations de R. Bourgi, un homme clé des réseaux Foccart, dans Laurent Valdiguié, «Bourgi : "J'ai vu Chirac et Villepin compter les billets"», Le JDD, http://www.lejdd.fr/Politique/Actualite/Lavocat-Robert-Bourgi-raconte-comment-il-a-convoye-jusqu-a-1-Elysee-les-milli ons-des-chefs-d-Etat-africains- interview-387001 
les paradis fiscaux (Européens ou non), les marchés de consommation industrielle ou des ménages qui se trouvent pour l'essentiel dans les pays développés.

\section{Un enjeu militaire essentiel}

Au cours de la période 2002-2011, l'Afrique du Nord a compté pour 2,7\% et l'Afrique subsaharienne pour 1,6\% des livraisons d'armes de la France, seul le Maroc est un client important de la France. La détresse financière des pays de la zone subsaharienne qui les saigne- ils figurent en bas du classement du développement humain -limite les possibilités d'exportations françaises. Celles-ci portent principalement sur l'équipement des forces terrestres et des unités d'élites et des navires patrouilleurs, et sont donc utilisées pour des opérations principalement dirigées contre les populations.

En dépit de la faible solvabilité de son marché militaire, le continent Africain possède une importance majeure pour la France au point qu'un rapport parlementaire pose la question «Le ministre de la défense, ministre de l'Afrique ? » et fournit les éléments pour répondre par l'affirmative $^{41}$. Depuis l'accession à l'indépendance des anciennes colonies, les liens militaires sont demeurés très denses. En mars 2015, la France comptait environ 7100 militaires engagés sur les théâtres d'opérations extérieures, dont plus de $70 \%$ en Afrique. Du point de vue de la défense, les partenariats passés avec les pays Africains, qui ont conduit à la présence de 30000 militaires présents dans 7 pays ${ }^{42}$ au début des années 1960 ont été progressivement reconsidérés au cours des années 2000. En 2013, la France

\footnotetext{
${ }^{41}$ Jean-Claude Guibal, et Philippe Baumel, Rapport de la mission d'information sur la stabilité et le développement de l'Afrique francophone, Assemblée Nationale, 6 mai 2015 n²746, 2015,p.138.

42Sénégal, Madagascar, République centrafricaine, Côte d'Ivoire, Tchad, Gabon et Djibouti.
} 
avait 52 attachés de défense et 24 accords de défense ou de coopération en Afrique pour un budget de plus d'un milliard d'euros par $a n^{43}$. Enfin, depuis le début des années 1960, La France est intervenue militairement à près de quarante reprises sur le sol africain dans les cinquante dernières années ${ }^{44}$.

La centralité du rôle de l'Afrique pour la France, en plus des intérêts économiques, demeure incontestable pour au moins quatre raisons : son statut international, les enjeux capacitaires et technologiques, et les ventes d'armes. D'abord, compte tenu des conflits qui déchirent le continent, la présence militaire de la France dans ces zones a une résonance mondiale et consolide son statut de membre permanent du CSNU. Elle conforte la préservation du 'rang mondial' de la France dont elle constitue avec la détention de l'arme nucléaire, l'autre pilier historique. Le représentant permanent de la France auprès des Nations unies rappelle que "L'Afrique représente $70 \%$ de l'activité du Conseil de sécurité des Nations unies, signe des crises qu'elle traverse. Dans la mesure où les résolutions sont présentées par les Etats membres, cela amène la France à être à l'origine de $60 \%$ des textes, concernant par exemple le Mali, la Côted'Ivoire, la RDC ou la RCA» ${ }^{45}$.

Sous l'angle croisé des enjeux capacitaires et technologiques, les opérations militaires sont également importantes. Elles permettent de déterminer ou de confirmer des développements technologiques. Les

43Jeanny Lorgeoux et Jean-Marie Bockel, " la présence de la France dans une Afrique convoitée ", Rapport d'information fait au nom de la commission des affaires étrangères, de la défense et des forces armées, Sénat, n 104, 9 octobre 2013.

${ }^{44}$ Direction de l'information légale et administrative, « Du Tchad au Mali : les interventions de l'armée française depuis 1981 ", Chronologies thématiques, mis à jour le 30 janvier 2013, http://www.viepublique.fr/chronologie/chronos-thematiques/du-tchad-au-mali-intervention s-armee-francaise-depuis-

1981.html. L'ONG Survie parle d'une cinquantaine d'interventions entre 1960 et 2009,cfSurvie, 2009 «Que fait l'armée française en Afrique ?",Dossier noir, n²3. ${ }^{45}$ Id., p.282 
interventions en Afghanistan et en Lybie ont montré la nécessité de disposer de drones de catégorie $\mathrm{MALE}^{46}$. De plus, l'interaction entre la DGA et les militaires engagés dans les conflits en Afrique permet d'améliorer le niveau de qualité et de performances des matériels ${ }^{47}$.

Ensuite, la multiplication des interventions militaires est nécessaire pour maintenir l'expertise et développer de nouvelles compétences, en particulier dans le domaine des opérations qualifiées de sécuritisation dans les zones massivement peuplées. Aux Etats-Unis, l'intérêt pour les doctrines de la "guerre urbaine" a fortement augmenté dans les années 1990, car les responsables savaient qu'ils auraient à faire face à des populations hostiles lors d'interventions extérieures, mais également face à d'éventuels "ennemis de l'intérieur"'18. La France est dotée d'une longue expérience en Afrique, mais, comme le rappelle un expert militaire de ce type d'opérations, "la guerre des Balkans [a été] un laboratoire pour la pensée stratégique" ${ }^{49}$. On peut ajouter que la professionnalisation des armées décidée par le président Chirac en 1996 avait pour objectif de préparer l'armée

\footnotetext{
${ }^{46}$ Xavier Pintat et Daniel Reiner, «Projet de loi de finances pour 2012 : Défense : équipement des forces», Sénat, 17 novembre 2011.

${ }^{47} \mathrm{Cf}$ Jean-Michel Fourgous, « la réactivité de la DGA montre l'intérêt de sa présence auprès des forces pour raccourcir la boucle du retour d'expérience », «Rapport sur le projet de loi de finances pour 2012 », Assemblée Nationale, n³805, 12 octobre 2011, p.43.

48 C. Serfati, La mondialisation armée, Textuel, La Discorde, 2001, Paris. Une étude consacrée à la 'lutte contre- subversive' considère que "La réflexion sur la subversion intérieure réapparaît au début des années 1970, parallèlement à l'émergence du 'problème de l'immigration » Mathieu Rigouste, "L'ennemi intérieur, de la guerre coloniale au contrôle sécuritaire», Cultures $\mathcal{E}$ Conflits, automne 2007, p.161.

${ }^{49}$ Michel Goya, "L'Armée française face au siège de Sarajevo, le combat urbain dans la pensée tactique et opérationnelle ",2012, http://www.defense.gouv.fr/irsem/publications/lettre-de-l-irsem/les-lettresde-l-irsem-

2012-2013/2012-lettre-de-1-irsem/lettre-de-1-irsem-n-5-2012/releve-strategique /dossier-special-les-20-ans-du-

siege-de-sarajevo-les-balkans-un-laboratoire-pour-la-pensee-strategique/l-arm ee-francaise-face-au-siege-de-

sarajevo-le-combat-urbain-dans-la-pensee-tactique-et-operationnelle.
} 
française à ces opérations 'urbaines'. C'est pourquoi "les Balkans [ont été] la matrice de la nouvelle Armée française professionnelle" ${ }^{\prime 50}$. Il va sans dire que la multiplication des interventions depuis 2007 augmente considérablement l'expertise des militaires. Elle suscite une certaine admiration de la part des responsables américains, qui considère qu'une raison du succès français tient à l'existence de corps historiquement centrés sur les opérations coloniales (marines et légionnaires) ${ }^{51}$.

Enfin, du point de vue industriel, il est non seulement indispensable que les équipements militaires soient testés par les armées nationales avant d'être proposés à l'exportation, mais leur utilisation dans des conditions réelles peut constituer un atout concurrentiel $^{52}$. Les interventions militaires représentent donc un moyen irremplaçable de promotion des exportations car il bénéficie auprès des pays clients d'un label 'combat-proven' (sic, testé en combat). Un rapport parlementaire destiné à renforcer un peu plus le dispositif de soutien aux exportations d'armes rappelle ainsi que "La qualité internationalement reconnue aux armées françaises est telle que les matériels opérés par ses hommes bénéficient, au travers des retours d'expériences (RETEX), d'un avantage souvent décisif en termes de crédibilité auprès des acheteurs potentiels" ${ }^{53}$. Même si on oublie le contexte d'enthousiasme qui prévalait à la fin 2014, l'argument peut jouer, et d'abord vis-à-vis des gouvernements Africains soucieux d'organiser le contrôle répressif de leurs populations. La France équipe les forces terrestres et les unités d'élites,

${ }^{50} \mathrm{Id}$.

51 Voir par exemple, Michael Shurkin France's War in Mali Lessons for an Expeditionary Army, RAND Corporation (RR-770), 2014, p.31.

52On peut ajouter que leur utilisation oblige à passer de nouvelles commandes aux industriels. Ainsi, quelque $20 \%$ des matériels terrestres de retour de l'opération Barkhane sont irrécupérables, Audition du général Pierre de Villiers, chef d'état-major des armées, Assemblée Nationale, 21 mai 2015.

${ }^{53}$ Nathalie Chabanne et M. Yves Foulon, Rapport d'information sur le dispositif de soutien aux exportations d'armement, Assemblée Nationale, n²469, 17 décembre 2014, p.68. 
auxquelles elle fournit par exemple des véhicules blindés ainsi que des frégates nécessaires à la lutte contre la piraterie.

En 2013, le PDG de Dassault, anticipant les contrats de vente du Rafale annoncés début 2015, avait déclaré que «l'opération an Mali a donc en une influence positive sur l'image du Rafale, comme avant celle effectuée en Libyes ${ }^{54}$. Cette position est partagée par les dirigeants des sept grands groupes industriels : "Équiper l'armée française qui dispose de matériels et systèmes parmi les plus performants au monde et qui démontrent régulièrement leurs qualités en opérations extérieures reste pour les États clients un gage de crédibilité renforcée et donc un avantage compétitif important ${ }^{\prime \prime 5}$. De fait, les dividendes des guerres sont d'ores et déjà engrangés. A la fin avril 2015, le ministre de la défense J.Y. Le Drian annonçait que l'industrie française avait déjà enregistré auprès des pays étrangers un montant de commandes de 15 milliards d'euros, un record historique $^{56}$

\section{L'ECONOMIE POLITIQUE DU MILITAIRE EN FRANCE}

Un point commun des théories marxistes de l'impérialisme est d'avoir analysé la configuration de l'économie mondiale comme le résultat combiné de la dynamique d'accumulation du capital et des relations au sein du système inter-étatique. La première guerre mondiale fut ainsi analysée comme le produit des interactions fatales entre la concurrence économique et les rivalités politiques. La disparition des guerres inter-impérialistes depuis 1945 et la domination militaire absolue des Etats- Unis dans le 'camp occidental' sont deux argument souvent entendus pour déclarer

${ }^{54}$ Éric Trappier, président-directeur général de Dassault Aviation, Assemblée Nationale, 11 septembre 2013.

55Jean Guisnel, "Les industriels de l'armement entendus à l'Élysée », Le Point.fr, 16 avril 2014.

56 Dominique Gallois, « 2015, année record pour l'industrie de l'armement de la France Le Monde, 30 avril 2014. 
l'obsolescence des théories de l'impérialisme. D’une part, ceci revient à ‘jeter l'enfant avec l'eau du bain' et considérer que l'absence de guerres intra-occidentales équivaut à la marginalisation du rôle de la puissance militaire dans les relations internationales, alors même que la militarisation de la planète connait à la fois une accélération et un élargissement (en particulier en Asie) depuis quelques années. D'autre part, il suffit d'observer les rivalités géopolitiques entre d'un côté les Etats-Unis et leurs alliés et d'un autre la Chine et la Russie pour mesurer les limites de ces arguments en ce qui concerne les grandes puissances impérialistes. De plus, la contraction de l'accumulation du capital dans les pays du centre et la crise de domination des Etats-Unis créent des ambitions dans d'autres pays ${ }^{57}$.

En tout cas, la période contemporaine de mondialisation du capital dominée par le capital financier n'invalide pas l'importance des facteurs politico-militaire au niveau mondial et national. La place qu'un pays occupe dans la 'mondialisation' dépend de la force de son économie mais également de sa puissance militaire ${ }^{58}$. Car les rapports sociaux - et le capital est d'abord un rapport social - sont politiquement construits et territorialement définis, dévaluant sérieusement les thèses sur l'avènement d'une classe capitaliste transnationale ${ }^{59}$. Autrement dit, l'agencement des relations entre le politique et l'économique diffère selon les pays, et il forme précisément la trame des particularités nationales. Sur ce plan, la France est de tous les pays développés, celui dont l'osmose entre le capitalisme et

${ }^{57}$ Certains reprennent aujourd'hui - quoiqu'avec prudence - le terme de sous-impérialisme pour qualifier les BRICS, qui avait été introduit par le marxiste Brésilien Marini en 1972, voir P. Bond, «BRICS and the tendency to sub-imperialism 10/04/2010, http:/ / www.pambazuka.net/en/category.php/features/91303

${ }^{58}$ Cette question, abordée par les historiens ( $\mathrm{P}$. Kennedy) et les chercheurs en économie politique internationale ne peut être abordée dans cet article.

${ }^{59} \mathrm{C}$. Serfati, 'The new configuration of the Capitalist class' in L. Panitch, G. Albo\& V. Chibber (eds), Registering Class, Socialist Register 2013, London: Merlin Press. 
l'Etat est à la fois la plus ancienne et la plus achevée. Cette configuration unique est la conséquence d'une sédimentation qui s'est progressivement formée sur les bases de l'absolutisme royal. La machine étatique puissante qu'il avait créée fut profilée et développée afin de répondre aux besoins du capitalisme. Cependant, la grande bourgeoisie française fut incapable de trouver dans la seule «action des 'lois naturelles' de la société, c'est-à-dire à la dépendance $d u$ capital ${ }^{60}$ les forces nécessaires à sa domination. A peine quelques décennies après la révolution française et la conquête de libertés démocratiques, il fallut rien de moins qu'un coup d'Etat organisé par L.N. Bonaparte et l'instauration d'une dictature pour consolider la domination du capital et assurer son expansion.

Pour la période contemporaine, il est nécessaire de prendre la pleine mesure de la mise en place et de la pérennité des institutions de la $\mathrm{V}^{\circ}$ république. Cela fait près de six décennies qu’un régime qualifié de "coup d'Etat permanent" par F. Mitterrand ${ }^{61}$, structure les relations entre le politique et l'économique. Si la marginalisation du rôle du parlement transforme nombre de revendications et d'actions en un problème politique directement dirigé contre le Président, on doit en même temps observer la résilience des institutions de la $\mathrm{V}^{\circ}$ république, évidemment renforcées par leur défense intégrale par le Parti socialiste dans le cadre de l'alternance politique. Ce régime institutionnel a produit en l'espace de quelques dizaines d'années une osmose plus poussée des élites des secteur public et privé, une densification de flux d'échanges financiers et commerciaux entre l'Etat et le capital, enfin une multiplication des réglementations qui limitent les solutions de continuité entre "l'Etat et le marché". Une analyse de l'histoire, de l'économie et de la sociologie de la plupart des grands groupes

${ }^{60}$ K. Marx, Le Capital, Livre 1, Chapitre XXVIII : «La législation sanguinaire contre les expropriés à partir de la fin $\mathrm{du} X \mathrm{XV}^{\circ}$ siècle. - Les lois sur les salaires». ${ }^{61}$ F. Mitterrand, Le coup d'Etat permanent, Plon, 1964, Paris. 
français qui dominent l'économie française ${ }^{62}$ révèlerait - à la manière dont le négatif 'révèle' la nature de la photographie - la multiplicité de leurs rapports structurels à l'Etat. Même les lois de 'décentralisation' votées à partir de 1982 ont renforcé la cohésion entre l'Etat et le capital. En plus d'aboutir à des développements excroissants de l'appareil étatique ${ }^{63}$, elles ont soit renforcé les liens existants entre l'Etat et les grands groupes des secteurs de l'eau et des travaux publics, soit créé de nouveaux liens entre l'appareil étatique ('décentralisé') et les groupes dans le secteur de la grande distribution (grâce aux autorisations d'implantation), dont les marchés tournés vers la consommation des ménages sont pourtant fort éloignés de la commande publique.

Les interactions entre le politique à l'économique sont donc solidement ancrées en France, et ceci explique pour une bonne part la place singulière qu'y tient le militaire. Si le militarisme a été une composante décisive de la formation des Etats modernes, comme C. Tilly et d'autres historiens l'ont noté, la solidité de son ancrage en France est un trait marquant.

Tous les 'voyants' du militarisme demeurent aujourd'hui obstinément allumés. Ils le sont sur le plan financier, même si les écheveaux budgétaires sont toujours difficiles à démêler. Justifiée par «un contexte stratégique qui se dégrades ${ }^{64}$, le projet de loi actualisant la programmation militaire pour les années 2015 à 2019 inscrit une augmentation de 3,8 milliards d'euros sur la période, soit des dépenses militaires qui atteindront

${ }^{62}$ Dans les secteurs de l'armement (et conjointement de l'aéronautique et l'espace), du nucléaire, du pétrole et de l'énergie, des transports et de l'automobile, des télécommunications, du bâtiment et des travaux publics.

${ }^{63}$ Est-ce parce qu'ils suspectent une certaine gloutonnerie que les médias parlent de 'millefeuille administratif"?

${ }^{64}$ Ministère de la défense, "Projet de loi actualisation de la programmation militaire 2014 / 2019. Dossier thématique », n.p. 
162,4 milliards d'euros sur la période ${ }^{65}$ militaire 2014-2019, sans compter les financements des opérations militaires sur le budget général ${ }^{66}$. Les voyants sont également très visibles sur la question des armes nucléaires, sur lequel le consensus gauche-droite s'est construit grâce au ralliement du PS à la 'force de frappe' en $1978^{67}$. La détention de l'arme nucléaire est tellement essentielle dans le maintien du siège de membre permanent du conseil de sécurité que le débat sur ces questions demeure encore très limité $^{68}$. Un spécialiste observe que «La France n'a réalisé aucune mesure de désarmement comme elle s'était engagée à le faire, en adoptant le Document final du TNP de 2010» ${ }^{69}$. Enfin, l'extension des mesures de contrôle sur la population résidant en France qui sera permise par la loi sur le renseignement votée en juin 2015, qualifiée de 'loi scélérate' par plusieurs organisations dont la Ligue des droits de l'homme, renforce la proximité entre le militaire et le sécuritaire. Cette proximité donne un nouveau souffle

${ }^{65} \mathrm{Il}$ faut d'autre part prendre en compte le fait que des dépenses qui devaient venir de recettes exceptionnelles (cessions immobilières, ventes de fréquences hertziennes) sont remplacées à hauteur de 5,2 milliards d'euros par des 'recettes certaines' pour les militaires et les industriels.

${ }^{66}$ Le financement des 'opérations extérieures' (OPEX) est assuré à concurrence de 450 millions d'euros par le ministère de la défense. Au-delà, le budget général acquitte la facture. En 2014, les OPEX ont coûté 1,12 milliard auquel il faut désormais ajouter les opérations de sécurité extérieure ('Sentinelle') estimées à 260 millions d'euros en 2015.

${ }^{67} \mathrm{~L}$ 'engouement pour les exportations d'armes commence avec l'élection de F. Mitterrand en 1981. Le "Programme commun de gouvernement Parti socialiste-Parti communiste » (27 juin 1972) annonce " la cessation de toute vente d'armes et matériels de guerre aux gouvernements colonialistes, racistes et fascistes » ainsi que «la stricte réglementation des ventes éventuelles d'armement à l'étranger » (souligné par moi, C.S.).

68 P. Quilès est une quasi-exception, voir Paul Quiles, Jean-Marie Collin, Bernard Norlain, Arrêtez la bombe! Cherche-midi, 2013, Paris. 28 février 2013. Un groupe de 10 députés et sénateurs note également que "Trop de civils et de militaires de hauts rangs voient le désarmement nucléaire en France comme un acte de trahison ou de déclin », Déclaration de 10 parlementaires français à la Conférence internationale sur l'impact humanitaire des armes nucléaires à Vienne, 9 décembre 2014.

69Jean -Marie Collin, « La France et sa bombe, Eclairages, GRIP, 27 février 2015. 
aux entreprises de l'armement et facilite l'émergence d'un système industriel militaire et sécuritaire ${ }^{70}$.

\section{LE MILITAIRE UN 'AVANTAGE COMPARATIF' DE LA FRANCE EN EUROPE?}

Il convient maintenant de noter que l'intensification des interventions militaires s'est produite alors que l'économie française faisait face à la crise de 2008. Depuis lors, les politiques conduites sous les présidences Sarkozy et Hollande, en dépit des dizaines de milliards d'euros versés aux entreprises, n’ont permis, ni de retrouver la croissance économique ni d'empêcher la hausse du chômage. Parmi d'autres signes alarmants, celui des 3,5 millions de personnes mal-logées (et 5 millions supplémentaires de personnes fragilisées par rapport au logement $)^{71}$ indique l'incapacité et l'absence de volonté de résoudre la « question du logement » qui accompagne l'histoire longue du capitalisme. L'industrie française perd des positions face aux autres pays industrialisés, comme l'atteste l'aggravation des déficits commerciaux avec la plupart d'entre eux ${ }^{72}$. L'affaiblissement de la base industrielle de la France qui a accéléré au cours des dernières années en est une des causes.

\footnotetext{
70 Voir C. Serfati, L'industrie française de défense, La documentation française, Paris 2014, chapitre 7

l'industrie de la sécurité ». Alors que, en 2013 le chiffre de l'industrie d'armement était de 15,1 milliards d'euros, celui de la 'sécurité nationale' (essentiellement financé sur fonds publics) était d'environ 8,5 milliards d'euros, et celui de la sécurité privée (entreprises et ménages) d'environ 6 milliards d'euros. La sécurité au sens large double donc le chiffre d'affaires de l'industrie de défense.

${ }^{71}$ Fondation Abbé Pierre, L'état du mal-logement en France, $20^{\circ}$ rapport annuel, 3 février 2015.

72 Voir par exemple la détérioration significative des échanges commerciaux avec l'Italie entre 2004 et 2014 sur toutes les gammes de produits industriels, Etudes et Eclairages, «Creusement du déficit bilatéral de la France avec l'Italie depuis dix ans» juin 2015, n58, http:/ / lekiosque.finances.gouv.fr
} 
La détérioration accélérée de la compétitivité de l'industrie et l'explosion de la dette publique (passée de 64,4\% du PIB fin 2007 à 95\% fin 2014) qui pompe des ressources croissantes au profit du capital financier ont des conséquences très importantes pour le statut de la France au sein de l'UE. La construction de l'UE a résulté de trois forces majeures : la dynamique d'internationalisation du capital, l'action des Etats puis le développement des institutions communautaires (Commission, Banque centrale Européenne, etc.). Du point de vue étatique, l'Allemagne et la France ont joué un rôle essentiel, combinant une forte interdépendance économique, mais également politique. Un certain partage des rôles a opéré : l'économie allemande a progressivement assis sa domination économique sur l'Europe, tandis que la France s'efforçait, grâce à son statut de puissance militaire mondiale, de faire intégrer les questions de défense dans l'agenda communautaire ${ }^{73}$. La montée en puissance au niveau communautaire des questions de défense, que le traité de Rome avait réservées aux Etats-national, est le fruit d'un patient travail des gouvernements français. Certes, l'intégration européenne dans les questions de défense pâlit par rapport à celle réalisée sur la monnaie, l'autre pilier constitutif de l'ordre régalien ; De plus, l'appétit pour le militaire est variable selon les pays. Dans ces conditions, l'impérialisme libéral' théorisé par R. Cooper permet de coupler l'intervention militaire et les missions humanitaires. Il permet un compromis entre les pays à forte industrie militaire (en particulier la France et la Grande-Bretagne) et les pays qui ont une longue tradition d'action humanitaire (en particulier les pays nordiques) $)^{74}$.

73 C. Serfati, «Imperialism in context: the Case of France», Historical Materialism, 2015, Volume 23, Issue 2.

${ }^{74} \mathrm{C}$. Serfati, Impérialisme et militarisme : actualité $d u 21^{\circ}$ siècle, Page 2, Lausanne, 2004. 
Depuis 2008, l'écart s'est creusé entre la situation de l'économie allemande et celle des autres pays Européens. La situation est particulièrement difficile pour la France, puisque le déséquilibre économique est de plus en plus flagrant au sein du 'couple' franco-allemand. L'incapacité des gouvernements français à respecter dans leur pays les plafonds de déficits et de dette publics qu'eux- mêmes préconisent pour les autres pays de l'Eurozone est un des motifs de tensions entre les deux pays.

L'activisme militaire de la France n'est certes pas susceptible de réduire l'écart économique avec l'Allemagne. En revanche, il consolide sa place de première puissance militaire au sein de l'UE. Ce rôle ne lui est pas contesté par la Grande-Bretagne, à la fois échaudée par le désastre en Irak et par la priorité qu'elle donne au rôle de l'OTAN dans la défense européenne. Il permet à la France tirer parti de cet 'avantage comparatif que constitue l'interventionnisme militaire. L'objectif ne saurait être la mise en place d'une défense 'unique', comme l'est aujourd'hui la monnaie, ou même simplement la création d'une défense unifiée. Il s'agit plutôt d'élever le niveau d'implication militaro-humanitaire de l'UE, et également de permettre aux pays « qui remplissent des critères plus élevés de capacités militaires et qui ont souscrit des engagements plus contraignants en la matière en vue des missions les plus exigeantes » d'établir une "coopération structurée permanente dans le cadre de $l^{\prime}$ Union ${ }^{75}$ et si possible d'en faire supporter les coûts par le budget communautaire $^{76}$. Dans ce cadre, l'objectif des gouvernements français est

75 Article 28A-6 du Traité Sur L'union Européenne.

${ }^{76}$ Actuellement le mécanisme Athena mutualise $10 \%$ du coût des opérations, le reste étant à la charge des pays intervenant. 
d'obtenir le soutien européen dans les opérations militaires en Afrique, et mieux encore de mener celles-ci sous la bannière européenne ${ }^{77}$.

Un autre objectif, important et symboliquement significatif, est de faire acter par l'UE l'exclusion des dépenses de défense (ou à minima de celles consacrées aux interventions militaires)du pacte de stabilité et de croissance. Cette vieille revendication, déjà émise dans les années $2000^{78}$, aurait le double avantage de desserrer l'étau du pacte de stabilité et de légitimer à l'échelle européenne l'exceptionnalisme militaire' de certains pays (au premier chef la France). Le gouvernement allemand n'est toutefois pas résolu à laisser la France tirer un trop grand parti de cet 'avantage comparatif ${ }^{79}$. L'action de la France s'inscrit cependant dans une perspective d'interventions militaires plus nombreuses dans le futur : ainsi que l'écrit un rapport d'un groupe de travail présidé par J. Solana, «la probabilité de conflits régionaux de grande ampleur au Moyen-Orient et la conséquences des guerres intra-étatiques en Afrique aux causes multiples, y compris de grandes insurrections politiques» ${ }^{80}$ constituera un facteur d'instabilité majeure pour l'Europe au cours des quinze prochaines années.

${ }_{77 C}$ 'est le cas en République centre-Africaine (2014). Au Mali, la mission EUTM fournit une aide à l'armée, tandis que la mission EUCAP soutient l'action de la gendarmerie nationale, la police et la garde nationale.

${ }^{78}$ Par exemple par madame Alliot-Marie, ministre de la défense, Délégation pour l'union Européenne, Assemblée Nationale, 15 février 2005.

79 Refusant les demandes du Président Hollande faites au sommet de Bruxelles (décembre 2013), la chancelière Merkel a déclaré «We cannot fund military missions in which we are not involved in the decision process" , Andrew Rettman, "France, Germany and UK show discord on EU defence ", EU.Observer, 20 décembre 2013, https:// euobserver.com/defence/122570 ${ }^{80}$ Javier Solana More Union In European Defence, Report Of A CEPS Task Force, Février 2015,p.10. 


\section{UN ENGRENAGE DANGEREUX \\ Rhetorique militaro-humanitaire...}

La prise de conscience du chaos qui bouleverse une partie de la planète et les menaces qui pèsent sur les intérêts géoéconomiques que la France retire de son statut de puissance mondiale expliquent l'accélération de l'interventionnisme militaire des présidences Sarkozy et Hollande. Les menaces provoquées par des groupes terroristes qui se réclament de l'islam sont mises en avant ${ }^{81}$. L'objectif affiché est d'aider les gouvernements en place à faire face à cette menace et si besoin dans le cas de l'Afrique d'aider ces pays à 'reconstruire un Etat de droit'.

Les analogies avec les doctrines du 'state'-building' (reconstruction des Etats) qui triomphèrent au cours des années 2000 aux Etats-Unis sont réelles. Les désastres produits par leur mise en œuvre par l'Administration Bush ont freiné leur succès aux Etats-Unis. Par une sorte de vases communicants, ils ont permis aux dirigeants Français de se présenter en hérauts de 'l'impérialisme libéral' préconisé par R. Cooper.

Ces arguments ne sont pas vraiment nouveaux, ils se trouvaient au cœur de l'idéologie de l'impérialisme du dix-neuvième siècle et de sa mission «civilisatrice». Aujourd'hui, on lui substitue fréquemment le terme « humanitaire », qualifié par R. Brauman de «neo-colonialisme bumanitaire» ${ }^{82}$. Les conditions et le langage ont bien sûr changé depuis un siècle et demi : la

${ }^{81}$ La justification donnée par le ministre de la défense est que « la mise en place d'un Etat terroriste à portée de l'Europe et de la France, dans les mains de groupes proche d'Al Qaïda au Maghreb islamique (AQMI) » cité par P. RIché, « Pourquoi la France entre en guerre au Mali », 12 janvier 2013, http:/ / rue89.nouvelobs.com/2013/01/12/ pourquoi-la-france-entre-en-guerreau-mali-238534.

82 «Les pièges de l'engagement humanitaire. Droit d'ingérence ou devoir $\mathrm{d}^{\prime}$ ingérence? Rencontre avec Rony Brauman», Politique autrement, Lettre $\mathrm{n}^{\circ} 20$, juin 2000, http://www.politique-autrement.org/Lettre-no-20-Lespieges-de-l-engagement-humanitaire-Droit-d-ingerence-ou-devoir-d-ingerence 
'bonne gouvernance' et la 'responsabilisation' (accountability) sont les paravents sémantiques contemporains, et la création de colonies et de 'protectorats' qui suivirent souvent les interventions étrangères au nom de la lutte contre le chaos, est bannie au profit de déclarations sur la 'responsabilité de la communauté internationale'.

Les analogies avec l'interventionnisme militaire passé ne doivent néanmoins pas masquer qu’il existe des différences significatives. Au dix-neuvième et début du vingtième siècle, les pays Européens allaient à la conquête de 'nouveaux' territoires. La conquête eut pour contrepartie le financement de mesures qui, bien que tronquées (par exemple dans le domaine de l'accès aux soins ou à l'éducation), avaient pour fonction de légitimer le contrôle de ces territoires et leur protection contre les menées des pays rivaux. Aujourd'hui, les interventions militaires ne visent ni à conquérir des territoires ni à subjuguer des populations en leur imposant l'ordre métropolitain, elles sont hautement sélectives et dépendent de l'importance des intérêts politiques et économiques en jeu. Il suffit de regarder le chaos créé par l'intervention en Afghanistan, en Irak ou en Libye et la fréquence des interventions françaises dans son 'pré-carré' Africain afin de sauver des dirigeants ou les remplacer par d'autres plus acceptables pour comprendre l'horizon temporel très court de ces interventions et le refus d'aider les populations à prendre leur sort en mains. La lourdeur du 'fardeau' supporté par les anciennes puissances coloniales explique les appels des dirigeantsFrançais et de l'ONU aux gouvernements des pays Africains pour qu'ils mettent en place une force militaire apte à faire régner l'ordre. Toutefois, la pérennité de la présence de l’armée française en Afrique montre qu’on en est très éloigné. 


\section{...ET SOUTIEN BIEN REEL AUX DICTATURES}

Les interventions militaires de la France en Afrique subsaharienne et peut-être prochainement une nouvelle fois en Libye, ne visent pas à s'attaquer aux causes profondes de la désagrégation politique qui frappe ces pays. Elles ne remettent pas en cause les pratiques kleptocratiques des dirigeants, dont l'enrichissement personnel transite par les banques françaises et Européennes pour se placer dans les paradis fiscaux, lorsqu'il n'est pas simplement investi en France ${ }^{83}$. Elles visent avant tout à confirmer que la France possède les moyens militaires de préserver l'emprise des grands groupes financiers, bancaires et industriels français sur les ressources de ces pays.

$\mathrm{Au}$ Moyen-Orient, dès la fin des relations privilégiées qu'elle entretenait le régime Irakien, la France a renforcé ses relations militaro-diplomatiques avec les monarchies pétrolières. Elle a signé des accords de coopération militaire et de sécurité avec le Koweit, (1993, renforcé en 2009), le Qatar (1994, complété en 1998) et les Émirats Arabes Unis (2010). Elle s'engage à s'engager militairement pour soutenir ces pays en cas «d'agression qui serait menée par un ou plusieurs Etats» ${ }^{84}$. Cette solidarité très forte a été extrêmement favorable aux ventes d'armes, puisque le Moyen-Orient figure de longue date à la première place pour les exportations françaises. En 2014, le total des contrats d'armes passés avec la seule Arabie saoudite a représenté $50 \%$ du total des contrats entrés en vigueur ${ }^{85}$. L'étude d'impact de l'accord avec les émirats souligne l'importance de cet accord «notamment en matière de formation au combat de nos

${ }^{83}$ Voir le dossier documenté établi par Xavier Harel et Thomas Hoffnung, Le scandale des biens mal acquis. Enquête sur les milliards volés de la Françafrique, Les Editions de la Découverte, Paris, 2011.

${ }^{84}$ Cette formule figure dans l'accord signé avec les Emirats d'Abu Dhabi, mais la formule est à peu près la même dans les autres accords.

${ }^{85}$ Délégué Général pour l'armement Laurent Collet-Billon, audition à l'Assemblée Nationale, 26 mai 2015. 
propres forces, de perspectives de débouchés pour nos industries de défense et de soutien à nos opérations extérieures ${ }^{86}$.

Alors qu'en 2011, le rapporteur sur un projet de loi portant sur la coopération militaire appelait à la vigilance » lors de la "conclusion on la rénovation de ces accords ${ }^{87}$, on a au contraire assisté sous les présidences de Sarkozy et Hollande, à une solidification des liens avec les monarchies du Golfe, et cela sans contrepartie sur les droits démocratique ou simplement humains dans ces pays.

Les dirigeants de la France espèrent retirer des bénéfices géopolitiques et économiques de cette connexion plus étroite avec les monarchies pétrolières. La volonté de vendre des armes, et plus largement d'ouvrir les marchés des pétromonarchies aux grands groupes français constitue un axe majeur de la "diplomatie économique" que L. Fabius a contribué à fortement développer. Ill'a résumé ainsi après l'annonce de 20 milliards d'euros de contrats passés avec l'Arabie saoudite : "Tout cela veut dire que nous avons eu raison de donner une forme de priorité à nos relations avec l'Arabie saoudite dans la région. Cette priorité, nous l'avons donnée d'abord pour des raisons politiques car l'Arabie saoudite est un partenaire de référence dans cet Orient troublé, avec lequel on peut travailler au règlement des crises" ${ }^{188}$.

L'appui inconditionnel aux régimes saoudiens et Qatariens, ainsi qu'au Général Al-Sissi en Egypte se traduit par un silence assourdissant des dirigeants Français sur la répression féroce et l'absence de droits démocratiques dans ces pays. Il est en conséquence' inutile de chercher

${ }^{86}$ Annexée au projet de loi présenté à l'Assemblée Nationale, 2 mars 2011.

87 Philippe Folliot, Projet de loi (N³194), adopté par le Sénat, autorisant l'approbation de l'accord entre le Gouvernement de la République française et le Gouvernement de la République du Cameroun instituant un partenariat de défense, Assemblée Nationale, 30 mars 2011.

88 «L'Arabie Saoudite envisage l'achat de deux réacteurs nucléaires EPR», http:/ / lexpansion.lexpress.fr/actualite-economique/l-arabie-saoudite-envisag e-1-achat-de-deux-reacteurs-nucleaires-epr_1693140.html\#XOXeZbtLRm5rSZT V. 
dans l'activisme militaro-diplomatique de la France observé depuis quelques années la volonté de soutenir les mouvements populaires qui embrasent les 'pays Arabes'. En ce domaine, la conduite de la France en Egypte et au Moyen-Orient est plutôt guidée par la "doctrine Alliot-Marie". Les armes livrées à ces pays par la France sont en effet utilisées contre les populations qui sont victimes de ces régimes dictatoriaux.

\section{Un engrenage dangereux}

Les choix militaro-diplomatiques des gouvernements français produisent un engrenage de subordination périlleux. Ils entrainent une implication croissante de la France dans des conflits militaires au Moyen-Orient en faveur des régimes saoudiens et Qatariens dont la politique extérieure et le militarisme sont directement orientés contre l'Iran. Au lendemain de l'annonce du contrat de ventes de Rafale à l'Egypte, le général Al-Sissi décidait une attaque aérienne contre les populations civiles en Libye, qualifiés de «crimes de guerre» par Amnesty International89. De même, l'Arabie Saoudite a également organisé une l'attaque aérienne au Yémen et des bombardements avec des munitions à fragmentation passibles d'accusation de crimes de guerre - et elle a annoncé en même temps une prochaine vague d'achats de matériels militaires à la France.

La vente d'armes sans aucune réserve aux pays du Moyen-Orient par la France agit donc comme un facilitateur des guerres meurtrières qui sévissent dans la région. Car les pays acheteurs sont decides utiliser ces armes, pour lesquelles ils ont d'ailleurs besoin de militaires formés. Le gouvernement français a par exemple montré sa disponibilité en signant un

89 Amnesty International, "Libya: Mounting evidence of war crimes in the wake of Egypt's airstrikes", 23 février

2015. 
accord pour la formation technique des pilotes et mécaniciens, mais plus largement pour l'instruction d'officiers du renseignement dans un accord séparé et confidentiel. Il y a donc lieu de prévoir que la hausse très importante des dépenses militaires des pays du Moyen-Orient, à laquelle l'industrie française participe en bonne place, se traduira par une violence plus forte que celle aujourd'hui observée.

Cette évolution engage la diplomatie de la France dans une mécanique incontrôlée. Il est en effet illusoire de penser que là où les Etats-Unis n'ont semé que le chaos et en subissent aujourd'hui les effets, les régimes qui luttent pour une forme de leadership régional apportent au contraire une quelconque stabilité. La décision de prendre position dans le conflit entre deux 'camps' (Monarchies pétrolières vs Iran) qui luttent pour renforcer leur influence régionale explique la position 'faucon' du gouvernement Français sur un accord concernant l'uranium Iranien, alors que l'Arabie saoudite annonce qu'elle cherchera à se doter de l'arme nucléaire si un accord est trouvé avec l'Iran90.

De plus, ces régimes instrumentalisent, lorsqu'ils ne les ont pas créés, les réseaux terroristes dont le gouvernement français fait ses ennemis principaux. La promiscuité du régime qatarien avec les groupes islamiques radicaux en Irak, Syrie et au Yémen est aussi notoire que l'est le financement d'Al Quaïda par l'Arabie saoudite (et les Etats-Unis) au cours des décennies 1990 et 200091. Ainsi que l'observe un ancien haut fonctionnaire du Ministère de la Défense, «L'Arabie saoudite, maison mère du Salafisme Jihadiste, a créé un Frankenstein dont elle redoute depuis quelques années l'effet boomerang. Aujourd'hui, les dirigeants du royaume

${ }^{90}$ David E. Sanger, « Saudi Arabia Promises to Match Iran in Nuclear Capability", New York Times, 13 mai 2015.

91Par exemple, Elizabeth Dickinson, « The Case against Qatar », Foreign Policy, 30 septembre 2014. 
semblent divisés sur la ligne à adopter»92. On pourrait ajouter au 'cercle de famille' les parrains qatariens de groupes d'islamistes radicaux en Syrie, et l'élargir encore plus en rappelant le rôle joué par l'oncle Sam93. Les rivalités pour la domination régionale entre les monarchies du golfe qui parrainent ces groupes armés sont ainsi exacerbées94.

\section{CONCLUSION}

Les déclarations de F. Hollande lors de sa visite du site de Dassault-Aviation citées en titre de cet article sont, somme toute, celles d'un président « normal », c'est-à-dire de quelqu'un qui s'inscrit dans la longue tradition de la $5^{\circ}$ république. Cependant, au-delà des différences de personnalités $^{95}$, le contexte a profondément changé depuis sa fondation par de Gaulle. D'une part, la condition supportée par des millions de salariés et leurs familles en raison de leur mise en concurrence par le capital a peu à voir avec celle qui prévalait lors des 'trente glorieuses' et la crise aggrave le sort subi par les jeunes des 'banlieues', plus encore lorsqu'ils sont issus de l'immigration. D'autre part, la centralité du militaire demeure, mais elle

${ }_{92}^{9}$ Pierre Conesa, «Non, les Occidentaux ne doivent pas intervenir militairement au Moyen-Orient», Diploweb.com, 15 septembre 2014.

${ }^{93}$ Voir l'article de Jason Burke, datant de 1999 ( !), « Frankenstein the CIA created », The Guardian, 17 janvier 1999.

${ }^{94}$ La grille de lecture d'un conflit sunnite-chiite est biaisée. Les rivalités pour la domination régionale entre $l^{\prime}$ Arabie saoudite et l'Iran conduisent celui-ci à établir des contacts étroits avec les Talibans (sunnites) car les deux parties sont inquiètes de la montée en puissance de l'ISIS, voir F . Peikar, "Whydid the Talibans go to Teheran ? », TheGuardian , 22 mai 2015 . La rivalité entre l'Arabie saoudite et le Quatar pour contrôler les groupes armés sunnites en Syrie et en Libye est très forte.

${ }^{95}$ Ainsi, Hollande se différencie de son prédécesseur, adepte des gesticulations qui ont par exemple jalonné l'épisode Libyen : libération des infirmières bulgares par Kadhafi, tente du " guide » plantée dans les jardins de l'hôtel Marigny, rôle de B.H. Lévy comme conseiller du président, voir par exemple Alfred de Montesquiou, 
s'inscrit aujourd'hui dans le cadre d'une détérioration accélérée de la situation géopolitique internationale et l'irruption de mouvements populaires qui minent les régimes situés dans la « zone prioritaire de la France », selon l'expression utilisée dans le Livre blanc 2013.

Les interventions militaires de la France ont pour objectif de conforter le statut international de la France. Celui-ci constitue un actif d'autant plus nécessaire à défendre que la position économique du capitalisme français se détériore, y compris au sein de l'Union européenne. Elles conduisent les gouvernements français à augmenter les budgets militaires et sécuritaires. Si ces évolutions se confirmaient, il resterait alors à mesurer les conséquences de l'écart croissant entre l'activisme militaire et l'état de l'économie et de la société française. 